\title{
THE CEOS RECOVERY OBSERVATORY PILOT
}

\author{
Steven Hosford ${ }^{\mathrm{a}}$, Catherine Proy ${ }^{\mathrm{a}}$, Alain Giros ${ }^{\mathrm{a}}$, Andrew Eddy ${ }^{\mathrm{b}}$, Ivan Petiteville ${ }^{\mathrm{c}}$, Chu Ishida ${ }^{\mathrm{d}}$, Francesco Gaetani ${ }^{\mathrm{e}}$, Stuart Frye ${ }^{\mathrm{f}}$, \\ Simona Zoffoli ${ }^{\mathrm{g}}$, Jens Danzeglocke ${ }^{\mathrm{h}}$
}

\author{
a Centre National d'Etudes Spatiales (CNES), 18 av Edouard Bélin, 31401 Toulouse \\ - steven.hosford@cnes.fr, catherine.proy@cnes.fr, alain.giros@cnes.fr \\ b Athena Global, La Frouste, 04150 Simiane-la-Rotonde, France - andrew.eddy@athenaglobal.com \\ ${ }^{c}$ European Space Agency (ESA), ESRIN, Via G.Galilei, CP 64, 00044 Frascati, Italy - ivan.petiteville@esa.int \\ d Japan Aerospace Exploration Agency (JAXA), Tsukuba Space Center 2-1-1 Sengen, Tsukuba, Ibaraki, 305-8505 Japan - \\ ishida.chu@jaxa.jp \\ ${ }^{\mathrm{e}}$ Group on Earth Observations (GEO) Secretariat, 7 bis, avenue de la Paix, Case postale 2300, CH-1211 Geneva 2, Switzerland- \\ fgaetani@geosec.org \\ ${ }^{\mathrm{f}}$ National Aeronautics and Space Administration (NASA), Godard Space Flight Center, 8800 Greenbelt Rd, Greenbelt, MD 20771, \\ United States - stuart.w.frye@nasa.gov \\ g Agenzia Spaziale Italiana (ASI), Via del Politecnico snc, 00133 Roma, Italy - simona.zoffoli@asi.it \\ h German Aerospace Center (DLR), Space Administration, Earth Observation, Bonn, Germany - jens.danzeglocke@dlr.de
}

\section{THEME: post-disaster recovery}

KEY WORDS: Earth Observation, post-disaster recovery, disaster management cycle

\begin{abstract}
:
Over the course of the last decade, large populations living in vulnerable areas have led to record damages and substantial loss of life in mega-disasters ranging from the deadly Indian Ocean tsunami of 2004 and Haiti earthquake of 2010; the catastrophic flood damages of Hurricane Katrina in 2005 and the Tohoku tsunami of 2011, and the astonishing extent of the environmental impact of the Deepwater Horizon explosion in 2009. These major catastrophes have widespread and long-lasting impacts with subsequent recovery and reconstruction costing billions of euros and lasting years. While satellite imagery is used on an ad hoc basis after many disasters to support damage assessment, there is currently no standard practice or system to coordinate acquisition of data and facilitate access for early recovery planning and recovery tracking and monitoring. CEOS led the creation of a Recovery Observatory Oversight Team, which brings together major recovery stakeholders such as the UNDP and the World Bank/Global Facility for Disaster Reduction and Recovery, value-adding providers and leading space agencies. The principal aims of the Observatory are to:

1. Demonstrate the utility of a wide range of earth observation data to facilitate the recovery and reconstruction phase following a major catastrophic event;

2. Provide a concrete case to focus efforts in identifying and resolving technical and organizational obstacles to facilitating the visibility and access to a relevant set of EO data; and

3. Develop dialogue and establish institutional relationships with the Recovery phase user community to best target data and information requirements;
\end{abstract}

The paper presented here will describe the work conducted in preparing for the triggering of a Recovery Observatory including support to rapid assessments and Post Disaster Needs Assessments by the EO community.

\section{INTRODUCTION}

Over the course of the past decade, the world has seen an unprecedented number of disasters, which are growing both in number and severity of impact. Large populations living in more vulnerable areas have led to record damages and substantial loss of life. In the last decade we have witnessed a series of catastrophic events: the deadly Indian Ocean tsunami and Haiti earthquake of 2004 and 2010; the catastrophic flood damages of Hurricane Katrina in 2005 and the Tohoku tsunami of 2011, and the astonishing extent of the environmental impact of the Deepwater Horizon explosion in 2009. These catastrophes have widespread and long-lasting impacts. Recovery after such disasters costs billions of dollars and lasts years. While satellite imagery is used on an ad hoc basis after many disasters to support damage assessment and track recovery efforts, there is currently no system to support the coordinated acquisition of data and facilitate its access. After catastrophic events of this magnitude, a coordinated approach would maximise the effectiveness of efforts and promote more widespread use of satellite data after smaller events by increasing the awareness of the benefits to be obtained through its use.

The concept of a "Recovery Observatory" was initially born from seeing huge quantities of Earth observation data that are made freely available following major disasters to many different users. These data are made available in an ad hoc 
manner and through a large variety of platforms, which means that their use both during and after the disaster (when this is authorised) is not optimised. Seeking to optimise the use of collected data, and understanding the value of implementing systematic observations for several years, CNES led the creation of a platform to gather and continue to make available Earth observation data following the devastating Haiti earthquake of January 2010. This project, named KalHaiti, has made great progress over the past four years, and has allowed CNES to identify lessons learned that underscore the importance of working before a catastrophic event takes place, and of working collectively, as a community. A single, coordinated approach supported by advance planning will greatly increase the efficiency and impact of actions taken following major events. These lessons have led to the creation of the Recovery Observatory Oversight Team (ROOT), which developed a proposal that was presented to the CEOS Plenary in November 2013, further developed in early 2014, and approved at the CEOS SIT-29 in April 2014.

A CEOS-led Recovery Observatory, a one-time demonstration in the 2014-2017 period, would allow the development of specific tools tailored to provide easy access to data over affected areas (pre-event data, response data and coordinated post event acquisitions). An organised repository, which serves as a discovery platform for data and products, would allow disaster managers to work in a known environment with advanced satellite products and promote their use to key user communities. Currently, while research shows that EO can be a valuable tool for recovery, DRM stakeholders do not have a clear example of how EO can be used to support Recovery efforts in an optimal scenario for data collection. Until now, sharing has not been put forward in a coordinated manner for any major catastrophes.

The main objective of the proposed Recovery Observatory is to facilitate access to data and products that support recovery from a catastrophic event. A subsidiary benefit for CEOS and satellite data providers will be the development of specific approaches and protocols for using satellite data to support disaster recovery, and raising awareness with the Disaster Risk Management community of the benefits of a satellite-based approach.

\section{LIFECYCLE OF THE RECOVERY OBSERVATORY}

\subsection{Overview}

The CEOS Recovery Observatory Pilot will cover a multi-year period, beginning with a preparatory phase, in which satellite agencies collaborate with international recovery stakeholders and value adders on rapid assessments and support to Post Disaster Needs Assessments (PDNAs) in order to prepare for the creation of a single large-scale Observatory. This preparatory phase has already begun. In addition to the preparatory phase, the Observatory includes:

- Triggering;

- Establishment; and

- Operations.

\subsection{Preparation}

The goal is to prepare the creation of a Recovery Observatory by working on all the required components in order to have all of them ready before entering "cold storage" and the beginning of the Observatory life-cycle. Numerous aspects have been identified:

- Technical components (website, database(s), processing chains)

- Data provision flows (data types, providers, licenses)

- Agreements with Users communities and partners (intermediary users, science users)

This phase should be closed after a "dry-run" formal exercise has been implemented successfully. The on-going support to PDNAs may serve as this dry-run phase. The preparation phase will take place in two-steps, through an iterative process, and will be conducted in close collaboration with CEOS WGISS.

Since April 2014, CEOS' WGISS has been conducting a summary review of requirements, and sounded out potential collaborating agencies for contributions in support of the Observatory. Contributing agencies within WGISS developed a plan for how data would be acquired, handled, stored and distributed in the event of an early trigger. This was put in place 1 January 2015. In parallel, WGISS developed the long-term architecture and for implementation in summer 2015.

The ROOT led parallel discussions with international stakeholders to ensure they are actively engaged and prepared to play a role in the actual triggering of the Observatory, by recommending to CEOS specific events where the scale and nature of the disaster lends itself to the creation of an Observatory. Through this process, UNDP, the Global Facility for Disaster Reduction and Recovery (GFDRR)/World Bank and UNOSAT have all joined the ROOT, and are working with the ROOT to establish a concise list of products useful to support early recovery and recovery implementation monitoring.

While it is impossible to determine the exact needs of a Recovery Observatory before an event takes place, there are a large number of requirements that are common to all recovery situations after major disasters. Baseline data requirements are required for both EO data and for derived products. These data and products support several different types of activity that change as the recovery phase, and lifetime of the observatory, progresses. These activities might include:

- Built area damage assessment (initial and later detailed)

- Natural resource and environment assessment

- Reconstruction planning support

- Reconstruction monitoring; and

- Change monitoring.

While there is no need for detailed acquisition plans before the triggering of the Observatory, the preparation phase should include the development of detailed EO data requirements for different types of Observatories: one for a major earthquake, one for a major volcanic eruption, one for a major flood and/or hurricane, and a tsunami. The ROOT has begun working on this in close coordination with recovery experts and value adding providers who are familiar with these needs. Some elements are quite common and involve situational awareness immediately after the event and for the damage assessment period, or involve baselining pre event and post event imagery in a single database. Other products might be specific to the disaster type historical flood analysis for future flood events, InSAR analysis for strain estimates after earthquake, etc. 


\subsection{Triggering}

The CEOS agencies supporting the Observatory are in many cases also members of the International Charter. It is clear that the Observatory is a useful complement to the Charter, and that for a disaster of the scope envisaged for the Observatory, it is highly unlikely that the Charter would not be activated. For this reason, Charter activation should be a pre-condition for the creation of an Observatory (no Observatory if no Charter activation). The plan however is to create only one Observatory created in the 2014-2017 period, with an evaluation 6 months after triggering to see what works and what does not and make recommendations to CEOS on next steps. In contrast, there is on average one Charter activation per week. Other criteria are necessary to determine when an Observatory is triggered.

The magnitude characteristic (exceptional event with far reaching impact in human and/or material terms) should be the first filter. The Observatory should be triggered when the catastrophic nature of the disaster and the scale of the anticipated recovery effort warrant it. This may be a CEOS assessment, or may be the result of a request to CEOS from an outside organisation that agrees to serve as a "filter" to assess which disasters may be the best candidates. Any of the members of the ROOT who choose to commit to the preparation of the Observatory could also suggest that the Observatory be triggered and call a teleconference to discuss why, according to predetermined criteria. One of these criteria could (and should) be advice or a request from a designated international DRM body. The ROOT would review the triggering scenarios, consider the main 'standard' requirements for the triggering, and establish whether there is critical mass within CEOS for the triggering.

A CEOS management level participation in the activation discussions is a good idea at the very least to associate this level with the decision. The CEO (CEOS Executive Officer) is the right person to manage this interface, however it should be clear that this representative is acting as a conduit for CEOS management (CEOS Chair/ CEOS SIT Chair) and has consulted with them bringing a CEOS management point of view to the $\mathrm{RO}$ activation decision. A draft triggering mechanism has been in place since January 2015, and at the time of drafting this paper, this was expected to be presented to the CEOS SIT meeting in April 2015.

\subsection{Establishment}

Once the RO is triggered, significant work is required to set up the Observatory, beyond simply tasking satellites and acquiring data. In order to make the Observatory an integral part of the recovery process, strong ties are required the national end user, who is ultimately responsible for the planning and implementation of all recovery efforts. Data and products collected and generated need to be geared to the needs established in coordination with the end user, based as a starting point on the generic products experimented with international stakeholders beforehand. An overview of the activities required to support the establishment of the Observatory include:

- Upload of Charter data and baseline products;

- $\quad$ Link to national end user;

- Development of RO activity grid;

- $\quad$ Protocols for RO operations;

- Value-adding coordination;

- Initial reporting; and
- $\quad$ Research and training \& capacity development.

\subsection{Operations}

There are numerous issues associated with the on-going operations of the Observatory. An initial list has been compiled to underscore the complexity of establishing and operating this test bed for a multi-year period engaging both international and local/national stakeholders from both the governmental and non-governmental sectors.

These issues include both institutional coordination and technical coordination.

\section{Institutional Coordination:}

- Liaison and development of institutional relationships, new relationship building;

- Support to national users (technical and policy), including new service development;

- Value-adding coordination and new product development; new value added partners;

- Regular Report of technical results to national institutions;

- Coordination and animation of new research activities around RO concept;

- Development of lessons learned and replication of RO model (in other countries).

Technical coordination:

- Management of the activity (applications) grid in coordination with the project manager;

- Management of the acquisition plan and tasking with CEOS agencies;

- Management of RO content (data, product, news, documents);

- Management of data processing;

- Technical reporting;

- Development of long-term plan for RO after initial set-up;

- Maintenance of the RO IT (corrections, evolutions).

\section{INSTITUTIONAL COOPERATION WITH RECOVERY STAKEHOLDERS}

\subsection{Overview}

While the long-term goal remains the establishment of a Recovery Observatory, there is much to be gained from smallerscale cooperation to develop institutional linkages between data suppliers, product generators and the user community. In order to develop a better understanding of what is possible and what is most useful, these partners have agreed to collaborate on rapid assessments for major disasters and Post Disaster Needs Assessments (PDNAs) in the first half of 2015, as work continues to prepare for the triggering of the Observatory.

\subsection{Rapid Assessments and Post Disaster Needs Assessments}

The ROOT is working closely with recovery stakeholders, including UNDP and GFDRR, for the implementation of satellite support to rapid assessments and PDNAs as a precursor to the establishment of the Recovery Observatory. The 
first case on which collaboration was considered was Typhoon Pam over Vanuatu in March 2015. CEOS agencies and the recovery stakeholders considered in detail the needs of the recovery community, particularly in establishing a rapid assessment of damage to housing, transport infrastructure and the agricultural sector after the storm. Severe damage was caused in the archipelago, but the vast areas and remote location of the islands did not allow for an easy on the ground assessment. The International Charter provided rapid information on damage to housing in key areas and blocked roads, but did not provide a comprehensive look at housing across all islands nor a detailed look at agricultural damage. At the time of drafting, CEOS was exploring with GFDRR specific products that might be generated to support the rapid damage assessment. These products could be baselined for future PDNA support to the Bank form the value-added sector.

\subsection{Lessons learned and promotion of the RO before triggering}

Regardless of the exact products to be generated in support of rapid assessment and PDNAs, there are many lessons learned about how to better collaborate to support early recovery through the initial collaboration between CEOS and the recovery stakeholders. A clear definition of needs and geographic areas of interest is required at the very outset, with associated products and data, and an assessment of pre and post crisis data availability to allow managers to determine the best course of action. Clear practices and standard operating procedures to support rapid assessments with satellite products are being drafted so that future collaboration will benefit from the lessons learned during the support to Typhoon Pam early recovery efforts.

\section{CONCLUSION}

Initial collaboration between CEOS and the Recovery stakeholder community has already proven fruitful in terms of identifying best practices and streamlining collaborative processes. The creation of a Recovery Observatory is expected to allow the development of standard products and practices that will greatly improve the efficiency of early recovery planning and recovery implementation monitoring. It is hoped that the Recovery Observatory pilot will lead to a comprehensive lessons learned exercise that may serve the recovery community in the coming years and lead to a legacy strategy that includes incorporating the most useful products and practices into common practice during recovery work after major disasters. This would lead to a sustained vision for satellite EO use in the recovery phase.

\section{ACKNOWLEDGEMENTS}

The authors wish to acknowledge the support received from the UNDP, UNOSAT and The Global Facility for Disaster Reduction and Recovery (GFDRR) in developing the concept of the Recovery Observatory and in defining its associated products. Since January 2015, these organizations have formally joined the ROOT and are participating fully in the implementation of the vision described here.

\section{REFERENCES}

Petiteville, I. (et alii.), "WCDRR and the CEOS Activities on Disasters”, unpublished paper submitted to ISRSE 36, March 2015. 